\title{
Boron-dipyrromethene-modified Olaparib Fluorescent Imaging Agent
}

National Cancer Institute

\section{Source}

National Cancer Institute. Boron-dipyrromethene-modified Olaparib Fluorescent Imaging

Agent. NCl Thesaurus. Code C133717.

A poly (ADP-ribose) polymerase type 1 (PARP1) fluorescence imaging agent based on the PARP1 inhibitor olaparib, in which the cyclopropane group of olaparib is replaced by the green fluorescent dye boron-dipyrromethene (BODIPY) fluorophore (FL), with potential fluorescent imaging activity. Upon administration of the fluorescent PARP1 inhibitor PARPi-FL, the olaparib binding moiety specifically targets and binds to PARP1, which is often overexpressed on cancer cells. Upon fluorescent imaging, the PARP1-expressing cancer cells can be visualized. PARP1, the nuclear enzyme that catalyzes posttranslational ADP-ribosylation of nuclear proteins, is activated by single-strand (SS) DNA breaks and overexpressed in certain tumor cells; it plays a key role in DNA repair, tumor cell resistance and survival. 